\title{
Steroid Induced Diabetes
}

\author{
Najla M Alduaiji* and Maha M Alduayji \\ Department of Medicine, College of Medicine, Qassim University, Qassim, KSA
}

*Corresponding author: Najla Mohammad Alduaiji, Department of Medicine, College of Medicine, Qassim University, Email: dr.duaiji@hotmail.com

\section{Review Article}

Volume 2 Issue 4

Received Date: August 27, 2017

Published Date: October 04, 2017

DOI: $10.23880 /$ doij-16000165

\section{Abstract}

Corticosteroids are the synthetic analogue of adrenal cortex hormones which were discovered in the 1940 s. Glucocorticoids are commonly used drugs in the inpatient and outpatient settings. Their use can cause steroidinduced hyperglycemia and steroid-induced diabetes. It is a common clinical problem with incidence as high as $46 \%$ of patients. It has preventable complications which increased both morbidity and mortality. Clinician should be alert to make proper screening especially for high risk population. The diagnosis of steroid induced diabetes is similar to diabetes mellitus. Management is challenging as there is limited evidence for variety of steroid regimens, variable glucose patterns and different patient factors. It starts with life style modifications to oral hypoglycemic agents and insulin. New medications for diabetes have very limited evidence to support their use. After stopping steroid, hyperglycemia usually normalized but may persist in some patients, so follow up is important to identify patients with persistent diabetes.

Keywords: Steroid; Glucocorticoid; Diabetes Mellitus; Hyperglycemia

\section{Introduction}

Corticosteroids are the synthetic analogue of adrenal cortex hormones which were discovered in the 1940s [1]. Since their discovery they were used for multiple dermatologic, ophthalmologic, rheumatologic, pulmonary, hematologic, and gastrointestinal disorders $[1,2]$. Its use for in-hospital patients is more than $10 \%$ [2]. On the other hand, as outpatient, it is mainly for respiratory illnesses and its duration is less than 5 days [2]. However, it was more than 6 months in $22 \%$ of the cases and greater than 5 years in $4.3 \%$ [2]. It has various systemic effects including; immunosuppressive, anti-inflammatory, anti-proliferative and vasoconstrictive effects [1]. Each corticosteroid has different glucocorticoid and mineralocorticoid potency [1]. It has a wide range of toxicity which seems to be related to average dose and cumulative duration of treatment but no specific threshold has been established [1].

One of the major side effects of corticosteroid is steroid induced hyperglycemia or diabetes mellitus in euglycemic patient or worsening of glucose control in diabetic patients which has been recognized for over 50 years [3]. It is one of the gradual side effects and it's the most common cause of drug induced hyperglycemia [4]. It has been also reported to cause prolonged preventable hospitalization, increased risk of infection, increased admission to intensive care units, increased hospital mortality, decrease function of the grafts in solid organ transplantation and in reported cases to reduce survival after hematological transplantation [35]. Its incidence is high as it reaches $46 \%$ of patients and it increases glucose level up to $68 \%$ in comparison to baseline [4]. It is also documented to occur in $12.7 \%$ of lupus patients treated with steroids, $14.7 \%$ of patients treated with steroids for respiratory diseases, and $23.5 \%$ of leprosy treated with steroids [3]. This effect results from inhibition of insulin release in pancreas, increase hepatic gluconeogenesis and decrease peripheral glucose uptake [2-4].

Different steroids have been used in different regimens and routes and subsequently they have different pattern on glucose curves [2]. For most 


\section{Diabetes and Obesity International Journal}

commonly used single dose of morning oral prednisone glucose peaks at 4-6 hours after prednisone intake and fall down late in the evening [4]. So, screening of glucose should be pre-lunch or dinner (afternoon or evening) $[2,3]$. Additionally, the treatment with long acting hypoglycemic agent will predispose patients to hypoglycemia at night and early morning [3].

\section{Screening and Monitoring}

Clinician should be alert to screen for pre-existing diabetes mellitus by requesting $\mathrm{HbA1c}$ before administering steroids [6]. It was suggested in a study to use stress test with $8 \mathrm{mg}$ dexamethasone before administration of the usual dose of steroid and follow up glucose, insulin and C-peptide to detect people at risk of steroid induced hyperglycemia but this study should be further tested to confirm its benefit [4]. High risk patients include; elderly, over weight and obese patients especially if it is truncal, patients with acanthosis nigricans, family history of diabetes, known to have impaired fasting glucose or impaired glucose tolerance, known cases of diseases or medications that impair glucose tolerance, in some ethnic groups and in those who previously had gestational diabetes, steroid induced hyperglycemia or who will be on high steroid doses and will use it for long duration [2-4,6].

Though family history of diabetes mellitus is a known risk factor for type II diabetes mellitus, it is association is weaker with steroid induced diabetes as one of the study pointed that individuals with steroid induced diabetes mellitus has less family history of diabetes when compared with those with type II diabetes mellitus [3].

If baseline glucose parameters are normal then home glucose monitoring is recommended at least once daily pre- or post- lunch or pre- dinner [2]. It is also recommended to measure blood glucose within the first 8 hours of the first dose of prednisone [1]. Others recommended monitoring for 48 hours of initiation of steroid irrespective of diabetes status since $94 \%$ of steroid induced hyperglycemia occurs in this period $[1,4]$. Guidelines also recommended monitoring blood glucose weekly for 4 weeks post-transplant then at 3 and 6 months then annually thereafter [1]. Once the diagnosis of steroid induced diabetes mellitus is made or in case of pre-existence diabetes, monitoring should be pre-meal and at bed time [2].

\section{Diagnosis}

Diagnosis of steroid induced diabetes mellitus is similar for patient who is not on steroid. It includes; fasting plasma glucose at or above $7.0 \mathrm{mmol} / \mathrm{L}$, HbA1c $\geq 6.5$ percent ( $48 \mathrm{mmol} / \mathrm{mol}$ ), a two-hour value in an OGTT at or above $11.1 \mathrm{mmol} / \mathrm{L}$, or a random plasma glucose concentration $\geq 11.1 \mathrm{mmol} / \mathrm{L}$ in the presence of symptoms $[1,3,4]$. However, use of fasting glucose as a sole parameter for diagnosis is discouraged as steroid effect mainly postprandial glucose $[3,4]$.

\section{Prevention and Treatment}

For prevention of steroid induced diabetes mellitus clinician should use the minimum effective dose and shorten the duration of treatment to the lowest that achieve treatment goals [1].

Initial step of management is lifestyle modifications including nutritional modifications and physical activity $[1,3]$. Target blood sugar is $4-7 \mathrm{mmol} / \mathrm{L}$ while fasting and 5-10 mmol/L for postprandial readings and $\mathrm{HbA1c}$ $<7 \%$ [1-4]. However, other accepted wider range 4-12 $\mathrm{mmol} / \mathrm{L}[2,6]$. Pharmacotherapy should be added in most cases whenever the target blood sugar is not met. The choice of medication depends on multiple variable which include; severity of hyperglycemia, type of steroid used (half-life, frequency and its potency) patient comorbidities and patient choice [6].

Oral hypoglycemic agents including sulfonylureas, meglitinides, metformin, pioglitazone, dipeptidyl peptidase-4 (DPP-4) inhibitors and sodium glucose transporters-2 (SGLT-2) inhibitors can be used in addition to glucagon like peptide-1 (GLP-1) agonists $[1,2]$. Although the evidence for the use of the last 3 medications for steroid induced diabetes is limited [2].

In patients with mild postprandial hyperglycemia, a trial of meglitinides immediately before the lunch can be used and their effect last for 4-6 hours with reassessment of the response [3,6]. If sulfonylureas are used, clinician should use the one correlated with steroid half-life [1]. For the commonly used once daily prednisone, short acting sulfonylurea like gliclazide and glyburide can be used and doses should be given with steroid [1,2]. For example, gliclazide can be initiated with $40 \mathrm{mg}$ and the dose can be increased if did not meet blood sugar targets to total morning dose of 240 $\mathrm{mg}$ and total daily dose of $320 \mathrm{mg}$ [2]. On the other hand, if it is twice daily prednisone or longer acting steroid (e.g. dexamethasone) is used and it induced diabetes mellitus, longer acting sulfonylurea like gliclazide twice daily, gliclazide MR or glimepiride are recommended [1]. For example, gliclazide can be initiated with $40 \mathrm{mg}$ and the dose can be increased if did not meet blood sugar targets to total dose of $160 \mathrm{mg}$ twice daily [2]. 


\section{Diabetes and Obesity International Journal}

Pioglitazone is weakly recommended for steroid induced diabetes considering that it needs few weeks for its maximum effect in addition for multiple contraindications [2]. It has also a risk of osteoporosis and fluid retention which might get worse with use of steroids [4].

Exenatide, GLP-1 agonist, where studied in randomized, double blinded, placebo-controlled study of 8 healthy individuals and showed prevention of glucocorticoid induced glucose intolerance [3,7]. Additionally, exenatides where found to be successful in treatment of glucocorticoid worsened diabetes in 4 reported cases of known diabetics $[4,8]$. But these studies are of limited number and further studies are needed to confirm such results.

DPP4, particularly sitagliptin, was tested in randomized double-blind study of 54 healthy individuals with high risk of development of steroid induced diabetes mellitus. It was found that it can ameliorate the diabetogenic effect by improving multiple aspects of pancreatic islet cells functions but it does not prevent it [9].

We do not have evidence to support use of sodium glucose transporters-2 (SGLT-2) inhibitors for steroid induced diabetes mellitus especially if one considers its side effects of genitourinary infections in susceptible patients [4].

It is unlikely to control blood glucose more than 15 mmol/L with oral hypoglycemic agents [1]. Thus, insulin is required in such cases [1]. It can be started with a dose of $0.15-0.3$ units $/ \mathrm{kg} /$ day [6]. Others recommend use of 10 units of insulin with daily increment of 10$20 \%$ especially for in-hospital patient [2].

If only morning dose of prednisone is used, fasting glucose might not be affected and hyperglycemia occurs late. Therefore, choosing intermediate acting insulin like NPH in the morning is reasonable in such cases $[1,2]$. However, if fasting glucose is also high or hyperglycemia is throughout the day, or if steroid is twice daily dose or longer acting steroid (e.g. dexamethasone) is used, twice dose of NPH or long acting insulin is recommended like glargine and detemir $[1,2]$. The addition of ultra-short insulin's (e.g. aspart or lispro) may be required pre-meal for post meal hyperglycemia [2,4]. Regular insulin can also be used for post-meal hyperglycemia particularly for patients with delayed gastric emptying and those who take snacks [4]. Basal bolus insulin's remain the most flexible option which include; basal insulin, prandial insulin and correction doses [3]. The calculated doses should be divided to $30 \%$ basal and $70 \%$ bolus $[4,6]$.
If a patient is known to have diabetes on insulin, it is suggested to increase insulin doses by $20 \%$ and adjust the dose according to response [4]. This increment could be required only for morning doses especially for those on premixed insulin's and using single morning dose of prednisone [2]. If close follow up is possible as in-hospital patient, insulin might be increased 2-4 units every 48 hours according to response [2]. Metformin can be added or titrated if no contraindication to minimize steroid dose though it is not a cornerstone in treatment of steroid induced diabetes [1-3].

In case of patient with significant hyperglycemia, especially if they are unwell and in-hospital, clinician may use IV insulin drip to control it before shifting to subcutaneous insulin [2-4]. The best medications that is studied and properly cover the hyperglycemic profile of steroid is insulin and thus we would recommend it over the others especially the new medications with absence of evidence to use it.

\section{Prognosis and Follow up}

Although hyperglycemia resolved after discontinuation of corticosteroid on most of the cases, some patients will have persistent diabetes mellitus especially those with risk factors for diabetes mellitus $[1,4]$. If steroid is tapered down gradually, the hypoglycemic agent should be reduced as well. There is some controversy with a percentage of reduction. Some reduce steroid and hypoglycemic agent in similar percent, for example, if prednisone is reduced by $5 \mathrm{mg}$ weekly from $20 \mathrm{mg}$ then hypoglycemic agents should be reduced by $20-25 \%$ simultaneously [2]. On the other hand, other reduce insulin by $50 \%$ of steroid percentage, for example, if steroid decreased by $50 \%$ insulin will be decreased by $25 \%$ [4]. On either way, clinician should follow glucose readings to guide him on further dosing of medications. Additionally, blood glucose should be monitored after cessation of steroid to diagnose persistent diabetes mellitus [2]. Thus, fasting glucose or oral glucose tolerance test is recommended after 6 weeks or HbA1c after 3 months [2].

\section{Conclusion}

Steroid induced diabetes mellitus is a common problem which causes major morbidities and mortalities. Identifying patients with risk factors and screening all patients for pre-existing type II DM are essential steps for early detection and proper management. Management is challenging and should be individualize for each patient depending on multiple variables including: severity of hyperglycemia, type of steroid used (half-life, frequency and its potency) 


\section{Diabetes and Obesity International Journal}

patient comorbidities and patient choice. Oral hypoglycemic agents particularly sulfonylurea can be used for hyperglycemia less than $15 \mathrm{mmol} / \mathrm{L}$. Newer agents have limited evidence to support their use. Insulin is the best medications that is studied and properly cover the hyperglycemic profile of steroid and thus we would recommend it over the others especially the new medications with limited evidence to support its use.

Insulin usually used for sever hyperglycemia ( $>15$ $\mathrm{mmol} / \mathrm{L}$ ) and in patients with pre-existing diabetes on insulin. Follow up is important for adjustment of medications based on blood sugar readings. Additional adjustment should be done with changing steroid doses or its discontinuation. Further studies are needed to explore the role of the new medications for steroid induced diabetes mellitus, as well as, guidelines for different steroid regimens are required with frequent update to meet the new therapeutic options.

\section{References}

1. Liu D, Ahmet A, Ward L, Krishnamoorthy P, Mandelcorn ED, et al. (2013) A practical guide to the monitoring and management of the complications of systemic corticosteroid therapy. Allergy, Asthma \& Clinical Immunology 9(1): 30.

2. Aled Roberts, June James, Ketan Dhatariya (2014) Management of Hyperglycaemia and Steroid (Glucocorticoid) Therapy. JBDS.

3. Hwang JL, Weiss RE (2014) Steroid-induced diabetes: a clinical and molecular approach to understanding and treatment. Diabetes Metab Res Rev 30(2): 96-102.
4. Tamez-Pérez HE, Quintanilla-Flores DL, RodríguezGutiérrez R, González-González JG, Tamez-Peña AL (2015) Steroid hyperglycemia: Prevalence, early detection and therapeutic recommendations: A narrative review. World Journal of Diabetes 6(8): 1073-1081.

5. Derr RL, Hsiao VC, Saudek CD (2008) Antecedent hyperglycemia is associated with an increased risk of neutropenic infections during bone marrow transplantation. Diabetes Care 31(10): 1972-1977.

6. Mills E, Devendra S (2015) Steroid-induced hyperglycaemia in primary care. London Journal of Primary Care 7(5): 103-106.

7. Van Raalte $\mathrm{DH}$, van Genugten RE, Linssen MM, Ouwens DM, Diamant M (2011) Glucagon-like peptide-1 receptor agonist treatment prevents glucocorticoid-induced glucose intolerance and islet-cell dysfunction in humans. Diabetes Care 34(2): 412-417.

8. Koji Matsuo, Takuo Nambu, Yuki Matsuda, Yugo Kanai, Shin Yonemitsu, et al. (2013) Evaluation of the Effects of Exenatide Administration in Patients with Type 2 Diabetes with Worsened Glycemic Control Caused by Glucocorticoid Therapy. Internal Medicine 52(1): 89-95.

9. Van Genugten RE, van Raalte $\mathrm{DH}$, Muskiet $\mathrm{MH}$, Heymans MW, Pouwels PJ, et al. (2014) Does dipeptidyl peptidase-4 inhibition prevent the diabetogenic effects of glucocorticoids in men with the metabolic syndrome? A randomized controlled trial. Eur J Endocrinol 170(3): 429-439. 